Elementary Theory of Finite Groups. By Louis Clark Mathewson, under the editorship of John Wesley Young. Boston, Houghton Mifflin, 1930. x+165 pp.

It is a fortunate fact that as mathematics progresses, large bodies of doctrine once on the outskirts of knowledge become organized in such a way that the ordinary student of college mathematics may procure a working acquaintance with them. This, for instance, has been the case with the calculus, much of the theory of equations, and projective geometry.

The theory of groups had long ago passed the stage of a set of isolated facts. It had become an organized structure. It was both inevitable and desirable that books should be written aiming at the exposition of group theory in a manner suitable for use in undergraduate classes. Dr. Mathewson has sought to do this. He has been faced with many problems, and it is to be expected that in seeking their solution he should not please everyone.

There is the problem of selection and arrangement of material. This has been admirably solved. After a few examples of groups, the elementary theory of permutation groups is given. This is followed by examples of an interesting nature, after which the general theory is renewed. There is then a chapter on abelian groups, one on abstract definitions, and one on isomorphisms and composition-series. Two chapters sketching important further developments conclude the book. The first of these on linear substitutions gives some proofs, the last, chiefly dealing with Galois theory of equations and the Lie theory, through the statements of definitions and theorems, conveys to the reader some idea of the richness of these fields.

The author of such a book must also answer two important questions. How rigorous and general should the proofs be? What type of notation should be used? One should not be dogmatic on these points. It is certainly not harsh criticism; it may even be praise to say that the author's answers to these questions differ widely from those of the reviewer. The reviewer believes that throughout our college texts too little regard for powerful and general notations and too much negligence of rigor are shown. In subjects like algebra and the calculus it may be argued that the manipulative use of the methods involved is so important that questions of rigor may, to a certain extent, be ignored. It would seem, however, that a study of group theory would be largely valuable as showing the student an example of rigorous thought seldom met with before graduate work, and as giving the student an understanding of the value of powerful concentrates in notation. It seems fair to believe that the author does not agree with this point of view. It is true that the proofs are such that a student, seeing the lack of rigor or of generality, is given enough hints to fill in the gap. The student would not, however, be led to note the lack. For instance, in proving that the number of transpositions into which a given permutation can be factored is always even or odd, a Vandermondian determinant is used without establishing the fact that it does not vanish-a point necessary to the argument. In proving that the order of a sub-group is a factor of the order of the group, the inductive step is omitted, though the form it would take has been made obvious. In the notation distinct letters are used where subscripts would seem more powerful; matrices are displayed rather than written in abbreviated form; and similar usages are followed throughout the text. 
The examples are chosen with great skill.

We should all welcome such a well thought out endeavor to make the theory of groups more teachable. But as long as mathematicians remain as happily diversified as at present some will think that such a book as that under consideration has made too great sacrifices of rigor and generality, while others would condemn the books the reviewer would like to see written as too abstract and their authors as too visionary. Nothing is more disputable than taste.

\section{H. INGRAHAM}

The Universe in the Light of Modern Physics. By Max Planck. Translated by W. H. Johnston. New York, Norton, 1931. 118 pp. \$2.00.

Planck (p. 8) insists that we are compelled to assume the existence of a world of reality behind the usual world of the senses and that progress in physics has been made only on this assumption. "But (p. 10) besides the world of sense and the real world, there is also a third world which must be carefully distinguished from these:- this is the world of Physics." The most far-reaching results of investigation (p.15) can be obtained only by following a road leading to a goal which is theoretically unobtainable; and that goal is the apprehension of true reality: as the view of the physical world is perfected, it recedes from the world of sense and approaches the world of reality. The principal intellectual tool for such progress is (p. 65) the hypothesis whose implications are drawn and subjected to the test of experimental verification.

In relativity and quantum theory (p. 18) we have two completely new ideas, but their advent means (p. 23) reconstruction, not destruction. The revolutionary consequences of these ideas are treated at some length, especially in their bearing (p. 28) on the meaning of a particle, on the nature (pp. 39-41) of the principle of uncertainty and (p. 45) the character of waves of probability, and finally on causality, determinism, indeterminism and free will. Whatever doubt (p. 59) these new ideas may have thrown on the question whether physics is really on the right path, Planck meets with the proposition (p. 60) that theory and experience were never before so closely linked in physics and with the confident prediction (p. 112) that the parts of classical theory which have had to be discarded will be supplanted by what is sounder and more adequate.

The new physics (p. 113) will give us an insight into the secrets of the theory of knowledge. A new need has arisen for ideas from philosophy. "For this reason (p. 113) a careful study of the views and ideas of our great philosophers might prove extremely valuable." "Modern Physics (pp. 113-4) impresses us particularly with the truth of the old doctrine which teaches that there are realities existing apart from our sense-perceptions, and that there are problems and conflicts where these realities are of greater value for us than the richest treasures of the world of experience."

These and similar propositions are urged with supporting reasons. The translation appears to be well done. The typography reaches a high order of excellence.

R. D. Carmichael 